\title{
Validation of Solar Sail Simulations for the NASA Solar Sail Demonstration Project
}

\author{
Alexander C. Braafladt ${ }^{1}$ \\ University of Minnesota, Minneapolis, MN, 55455 \\ Alexandra B. Artusio-Glimpse ${ }^{2}$ \\ Rochester Institute of Technology, Rochester, NY, 14628 \\ Andrew F. Heaton ${ }^{3}$ \\ NASA Marshall Spaceflight Center, Huntsville, AL, 35812
}

\begin{abstract}
NASA's Solar Sail Demonstration project partner L'Garde is currently assembling a flightlike sail assembly for a series of ground demonstration tests beginning in $\mathbf{2 0 1 5}$. For future missions of this sail that might validate solar sail technology, it is necessary to have an accurate sail thrust model. One of the primary requirements of a proposed potential technology validation mission will be to demonstrate solar sail thrust over a set time period, which for this project is nominally 30 days. This requirement would be met by comparing a L'Garde-developed trajectory simulation to the as-flown trajectory. The current sail simulation baseline for L'Garde is a Systems Tool Kit (STK) plug-in that includes a customdesigned model of the L'Garde sail. The STK simulation has been verified for a flat plate model by comparing it to the NASA-developed Solar Sail Spaceflight Simulation Software (S5). S5 matched STK with a high degree of accuracy and the results of the validation indicate that the L'Garde STK model is accurate enough to meet the potential future mission requirements. Additionally, since the L'Garde sail deviates considerably from a flat plate, a force model for a non-flat sail provided by $L$ 'Garde sail was also tested and compared to a flat plate model in S5. This result will be used in the future as a basis of comparison to the non-flat sail model being developed for STK.
\end{abstract}

\section{Nomenclature}

$=$ sail area

$=$ back non-Lambertian coefficient ${ }^{1,9}$

$=$ front non-Lambertian coefficient ${ }^{1,9}$

$=$ force component along sail $\mathrm{x}$ body axis

$=$ force component along sail $\mathrm{z}$ body axis

$=$ solar pressure at one astronomical unit (AU)

$=$ reflection coefficient $^{1}$

$=$ propulsive reflectivity coefficient ${ }^{9}$

$=$ fraction of specular reflection ${ }^{1}$

$=$ back surface emissivity ${ }^{1,9}$

$=$ front surface emissivity ${ }^{1,9}$

$=$ sun incidence or pitch angle ${ }^{1,9}$

\footnotetext{
${ }^{1}$ Summer 2013 Intern, EV42/Guidance, Navigation, and Mission Analysis Branch, NASA Marshall Spaceflight Center

${ }^{2}$ Summer 2014 Intern, EV42/Guidance, Navigation, and Mission Analysis Branch, NASA Marshall Spaceflight Center

${ }^{3}$ Senior Aerospace Engineer, EV42/Guidance, Navigation, and Mission Analysis Branch, NASA Marshall Spaceflight Center
} 


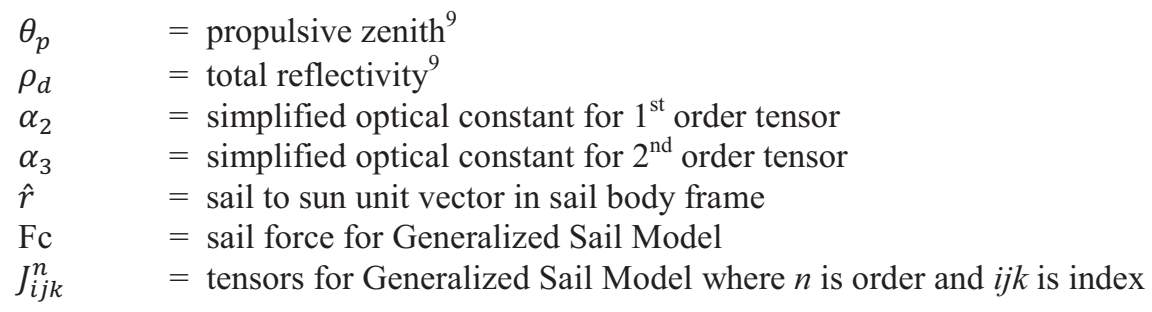

\section{Introduction}

$\mathrm{A}$ s future missions studying the solar system are designed based on scientific objectives in areas such as planetary science, heliophysics, and interplanetary exploration, solar sails provide the ability to reach some orbits and locations either difficult to achieve with conventional chemical propulsion, or at a reduced overall cost. Solar sails produce thrust solely through the use of Solar Radiation Pressure (SRP) from the Sun, giving them the ability to operate in space with continuous thrust for long periods of time and without the need for propellant. The fundamental process from which this thrust is derived is the momentum transfer of photons reflecting off the sail surface. This process has been studied extensively, especially since it disturbs the orbits of all spacecraft exposed to the sun. Many solar sail studies and some planned missions have resulted from the understanding of this readily available source of thrust. At NASA specifically, these include the proposed but ultimately scrapped Halley's Comet rendezvous in the $1970 \mathrm{~s}^{1,2}$ and the use of Mariner 10's solar panels as solar sails for attitude control. ${ }^{3}$ More recently, NASA funded ground demonstrations by ATK and L'Garde of 20-meter, fully-integrated sail systems in $2005^{4,5}$ (as seen in figure 1), and also NanoSail-D, a 3U Cubesat that deployed a 10 square meter sail in 2011 to test the use of a small sail for Low Earth Orbit (LEO) end-of-life de-orbit. ${ }^{6}$ Also, the MESSENGER mission, launched in 2004 and continuing operation today, used SRP for trajectory and attitude control in reaching orbit around Mercury. ${ }^{7}$ The Japanese Aerospace Exploration Agency (JAXA) also has been involved in developing and demonstrating the use of solar sails, and in 2010 deployed and launched the solar sail thrust demonstration spacecraft IKAROS to Venus. ${ }^{8}$ Even with the benefits of using a propellant-less source of continuous in-space thrust, because of the difficulties involved in manufacturing solar sail thin films and structures, along with the risks in deploying such huge structures in space and also those inherent in using an untested technology, solar sails currently still reside at the "proof-of-concept" level for main spacecraft propulsion.
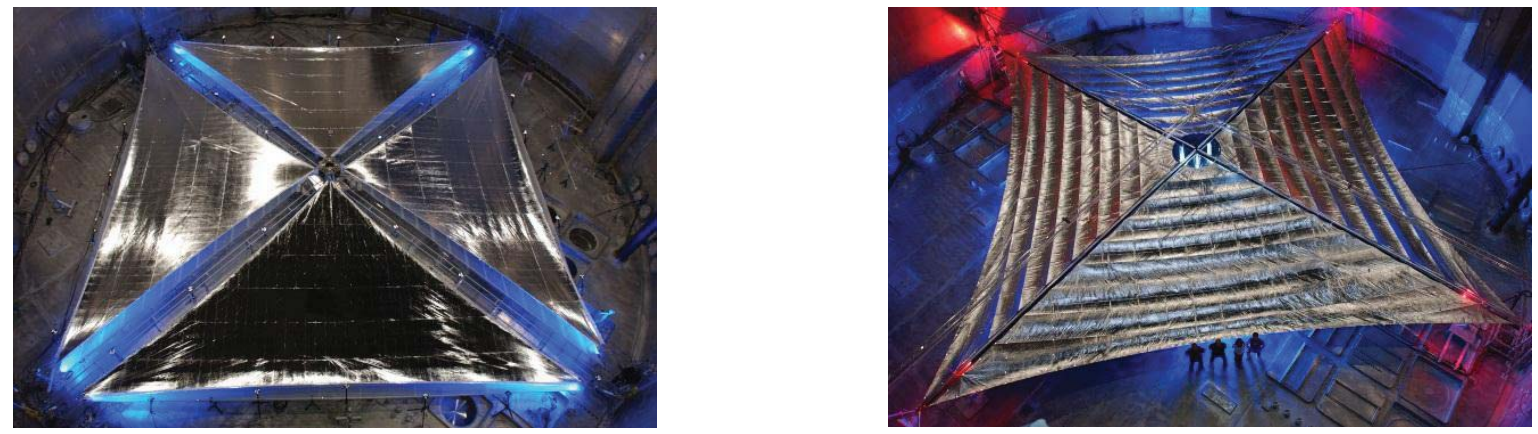

Figures 1a and 1b. ATK (left) and L'Garde (right) ground demonstration sail systems in the Glenn Research Center Plum Brook space environment simulation chamber. ${ }^{4,5}$

Following the success of NanoSail-D and the ATK and L'Garde ground sail system demonstrations, NASA is funding the Solar Sail Demonstration Project as part of the Technology Demonstration Mission (TDM) program with sail manufacturer L'Garde. For this project, a set of requirements was established to support future Missions. The requirements included that a future mission of the sail be able to perform "a 30-day maneuver sequence [...] with less than 20 percent error by comparison with the predicted final velocity and position relative to Earth." "For a future mission, this requirement is planned to be met by comparing the as-flown trajectory to a simulated trajectory based on the projected performance of the sail. The simulation L'Garde will use for this purpose is an AGI Systems Tool Kit (STK) "plug-in" model of the sail. At the time of the writing of this paper, the STK plug-in consisted of a

\footnotetext{
* Mission requirements from Solar Sail Demonstration Project peer review May 2013.
} 
flat plate sail with the L'Garde optics model. In the future, L'Garde plans to improve the solar sail model to include the model of their sail that incorporates shape. In this paper, we validate the flat plate model of L'Garde by comparing it to with the NASA-developed Solar Sail Spaceflight Simulation Software (S5). ${ }^{9}$ We also use the shaped sail model in S5 to model the shape of the L'Garde sail and compare it to a flat plate model for future reference.

\section{Technical Approach}

The comparison between the L'Garde STK simulation and the corresponding simulation in S5 encompasses a number of factors. Each software tool incorporates different options for gravity models, SRP models, integrators, propagators, coordinate systems, and attitude representation.

\section{A. L'Garde STK Simulation}

The solar sail trajectory was simulated by L'Garde using STK Astrogator with a plug-in developed by Applied Defense Solutions (ADS) containing the solar sail propagator. Included along with the plug-in are the STK high precision orbit propagator (HPOP v8.1.1), which uses a gravity model of Earth to degree and order 21, along with a point mass model of the Sun, and a point mass model of the Moon. The SRP force model used was that developed by Derbes and Lichodziejewski ${ }^{10}$ through tests on prototype sail materials. This model describes the forces in terms of a body coordinate frame as shown in Figure 2 .

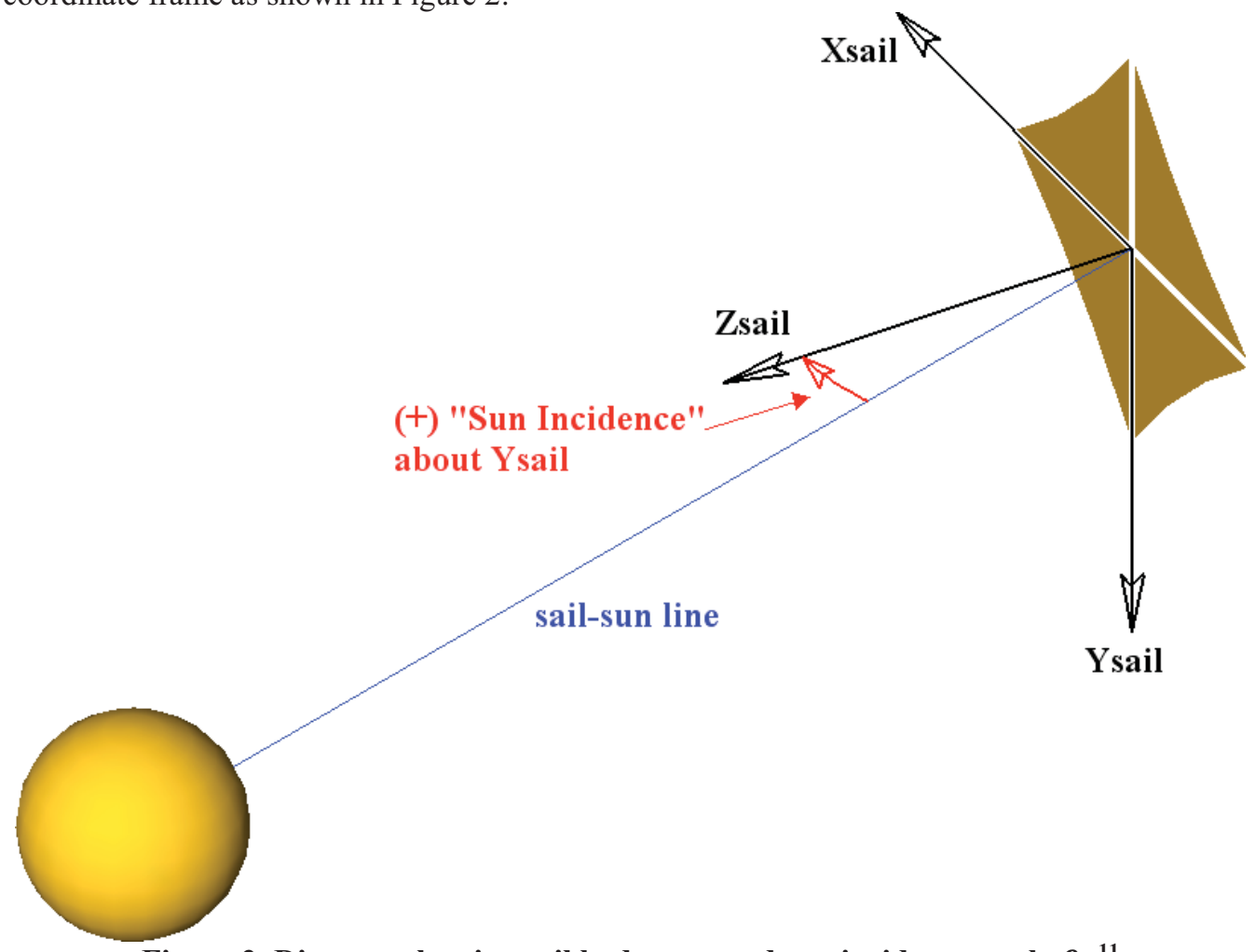

Figure 2. Diagram showing sail body axes and sun incidence angle $\theta_{i} \cdot{ }^{11}$

The force developed by the sail from the SRP is given in the transverse and normal directions in Eq. (1) and Eq. (2) respectively below, both of which are functions of sun incidence angle $\theta_{i} . P$ is the solar pressure at $1 \mathrm{AU}$ of $4.511918 \times 10^{-6} \mathrm{~Pa}$ and the other constants are defined in the appendix.

$$
\begin{gathered}
F_{x}=P A \cos \left(\theta_{i}\right) \sin \left(\theta_{i}\right)-R_{p} P A \cos \left(\theta_{i}\right) \sin \left(\theta_{p}\right) \\
F_{z}=-P A \cos ^{2}\left(\theta_{i}\right)-R_{p} P A \cos \left(\theta_{i}\right) \cos \left(\theta_{p}\right)-\left(1-\rho_{d}\right) P A \cos \left(\theta_{i}\right)\left(\frac{\varepsilon_{f} B_{f}-\varepsilon_{b} B_{b}}{\varepsilon_{f}+\varepsilon_{b}}\right)
\end{gathered}
$$

The attitude of the sail is defined as in Derbes, ${ }^{11}$ where top, sun incidence, and flat spin angles are a 3-2-3 Euler angle sequence about the body axes as are shown in Figure 2, starting from an initial orientation with the $\mathrm{z}$ body axis pointed at the Sun and the $\mathrm{x}$ body axis parallel to the ecliptic plane. Also, the propagation in STK used the RungeKutta $7 / 8$ (Fehlberg) method. 
For this study, the mass of the L'Garde sailcraft was assumed to be $34.641 \mathrm{~kg}$, resulting in a characteristic acceleration of $0.289 \mathrm{~mm} / \mathrm{sec}^{\wedge} 2$.

\section{B. S5 Simulation}

The simulation of the relevant part of the Sunjammer mission using S5 allowed a selection of different options. The gravity model available in S5 was the J2 model of Earth, along with point mass gravity models of the Sun and the Moon. The SRP models available in S5 are both the McInnes model ${ }^{1}$ and the Derbes and Lichodziejewski model as above. In terms of similar body axes as in Figure 2, Eq. (3) and (4) give the McInnes model force in the transverse and normal directions. S5 uses a value of SRP at $1 \mathrm{AU}$ of $4.571176 \times 10^{-6} \mathrm{~Pa}$ in the McInnes SRP model, the forces are again functions of sun incidence angle $\theta_{i}$. In order for the McInnes model to match the Derbes model, we added a gain constant $\mathrm{k}$ to the $\theta_{i}$ in the sine term of Equation (3) to make it match $\theta_{p}$ in the second term of Equation (1) (see Appendix for value of k). In the Derbes SRP model in S5 the value of SRP at 1 AU is consistent with that used in STK.

$F_{x}=-P A(1-\tilde{r} s) \cos \left(\theta_{i}\right) \sin \left(k \theta_{i}\right)$

$F_{z}=-P A(1-\tilde{r} s) \cos ^{2}\left(\theta_{i}\right)-P A B_{f}(1-s) \tilde{r} \cos \left(\theta_{i}\right)-P A(1-\tilde{r})\left(\frac{\varepsilon_{f} B_{f}-\varepsilon_{b} B_{b}}{\varepsilon_{f}+\varepsilon_{b}}\right) \cos \left(\theta_{i}\right)$

The sail attitude was defined to match that in the L'Garde STK plugin, and the propagation in S5 used the RungeKutta 7/8 (Fehlberg) method also.

\section{Comparison}

After propagating from the same initial state using their respective force models, the final state at the end of the 30 day maneuver sequence was output from both STK and S5 for comparison. Values of percent error were then calculated from the two state vectors to be consistent with the requirement as stated. Two different methods of calculating percent error were used for comparison as a final decision on the method of percent error calculation for the mission requirement (less than twenty percent error on position and velocity) has not yet been made. The first method uses vectors in the ICRF ${ }^{12}$ coordinate frame and calculates percent error as in Eq. (5) and Eq. (6).

Position Percent Error $=100 * \frac{\left\|\vec{r}_{S 5_{2}}-\vec{r}_{S T K_{2}}\right\|}{\left\|\vec{r}_{S T K_{2}}\right\|}$
Velocity Percent Error $=100 * \frac{\left\|\vec{v}_{S 5_{2}}-\vec{v}_{S T K_{2}}\right\|}{\left\|\vec{v}_{S T K_{2}}\right\|}$

Here $\vec{r}_{S 5_{2}}$ is the vector to the final position of the sail calculated using $\mathrm{S} 5, \vec{r}_{S T K_{2}}$ is the vector to the final position of the sail calculated using the STK plug-in, $\vec{v}_{S 5_{2}}$ is the velocity of the sail at the end of the maneuver sequence calculated using S5, and finally, $\vec{v}_{S T K_{2}}$ is the velocity of the sail at the end of the maneuver calculated using STK.

The second method for calculating the percent error between the two trajectories is again in the ICRF frame; however, the error values are instead calculated as in Eq. (7) and Eq. (8).

Position Percent Error $=100 * \frac{\left\|\Delta \vec{r}_{S 5}-\Delta \vec{r}_{S T K}\right\|}{\left\|\Delta \vec{r}_{S T K}\right\|}$
Velocity Percent Error $=100 * \frac{\left\|\Delta \vec{v}_{S 5}-\Delta \vec{v}_{S T K}\right\|}{\left\|\Delta \vec{v}_{S T K}\right\|}$

In these two equations, $\Delta \vec{r}_{S 5}=\vec{r}_{S 5_{2}}-\vec{r}_{S 5_{1}}$ and $\Delta \vec{r}_{S T K}=\vec{r}_{S T K_{2}}-\vec{r}_{S T K_{1}}$ are the differences between initial and final position in S5 and STK respectively, and $\Delta \vec{v}_{S 5}=\vec{v}_{S 5_{2}}-\vec{v}_{S 5_{1}}$ and $\Delta \vec{v}_{S T K}=\vec{v}_{S T K_{2}}-\vec{v}_{S T K_{1}}$ are the differences in initial and final velocities. Also, note that $\vec{r}_{S 5_{1}}=\vec{r}_{S T K_{1}}$ and $\vec{v}_{S 5_{1}}=\vec{v}_{S T K_{1}}$, as both sail simulations start from the same initial state.

These two error methods were also used to estimate the effects of attitude error on the ability to achieve the mission requirement of $20 \%$ error on position and velocity. For this estimation the first two angles in the 3-2-3 Euler angle sequence (top spin about the $\mathrm{z}$ body axis and sun incidence about the $\mathrm{y}$ body axis respectively) were varied $\pm 2^{\circ}$ in the S5 simulation. This variance is from the trim conditions of $35.5^{\circ}$ sun incidence and $10^{\circ}$ top spin. 


\section{Results and Discussion}

The comparison between the L'Garde STK and the S5 simulations using the percent error calculations as above resulted in the two simulations matching to a high degree of accuracy. Three different test cases were used to assess the performance of the trajectory analysis. An additional test measured the effect on performance of the maximum allowable attitude error, and a final comparison between the flat plate and shaped sail models indicates an impact of sail shape on trajectory.

\section{A. Flat Plate Comparisons}

The first test case was propagation using both sets of software with no SRP forces acting on the spacecraft in either simulation for the maneuver sequence length of thirty days. This was done to define the mismatch between the models in terms of the effects of differences in simulation such as integration, propagation, and gravity/force field models without the sail. The results of this comparison are shown in Table 1 in the first row for both methods 1 and 2 ("Spacecraft (No SRP)"). These percent error values are in the range of approximately $0.1 \%$ error on both position and velocity across error calculation methods. This shows decent agreement in general between the two propagators, and while the accuracy could be improved, it is sufficient for the purposes of validating the L'Garde model at this stage of the mission design process.

Table 1. Results of the comparison between STK and S5 in percent error on position and velocity.

\begin{tabular}{|l|l|l|}
\hline (Method 1) & Position Percent Error & Velocity Percent Error \\
\hline Spacecraft (No SRP) & $0.0158 \%$ & $0.0487 \%$ \\
\hline Without Sail & $40.2812 \%$ & $195.1938 \%$ \\
\hline Sail & $0.0854 \%$ & $0.3012 \%$ \\
\hline (Method 2 $\Delta \mathbf{r}, \Delta \mathbf{v})$ & Position Percent Error & Velocity Percent Error \\
\hline Spacecraft (No SRP) & $0.0222 \%$ & $0.0921 \%$ \\
\hline Without Sail & $63.5495 \%$ & $64.0808 \%$ \\
\hline Sail & $0.1348 \%$ & $0.0989 \%$ \\
\hline
\end{tabular}

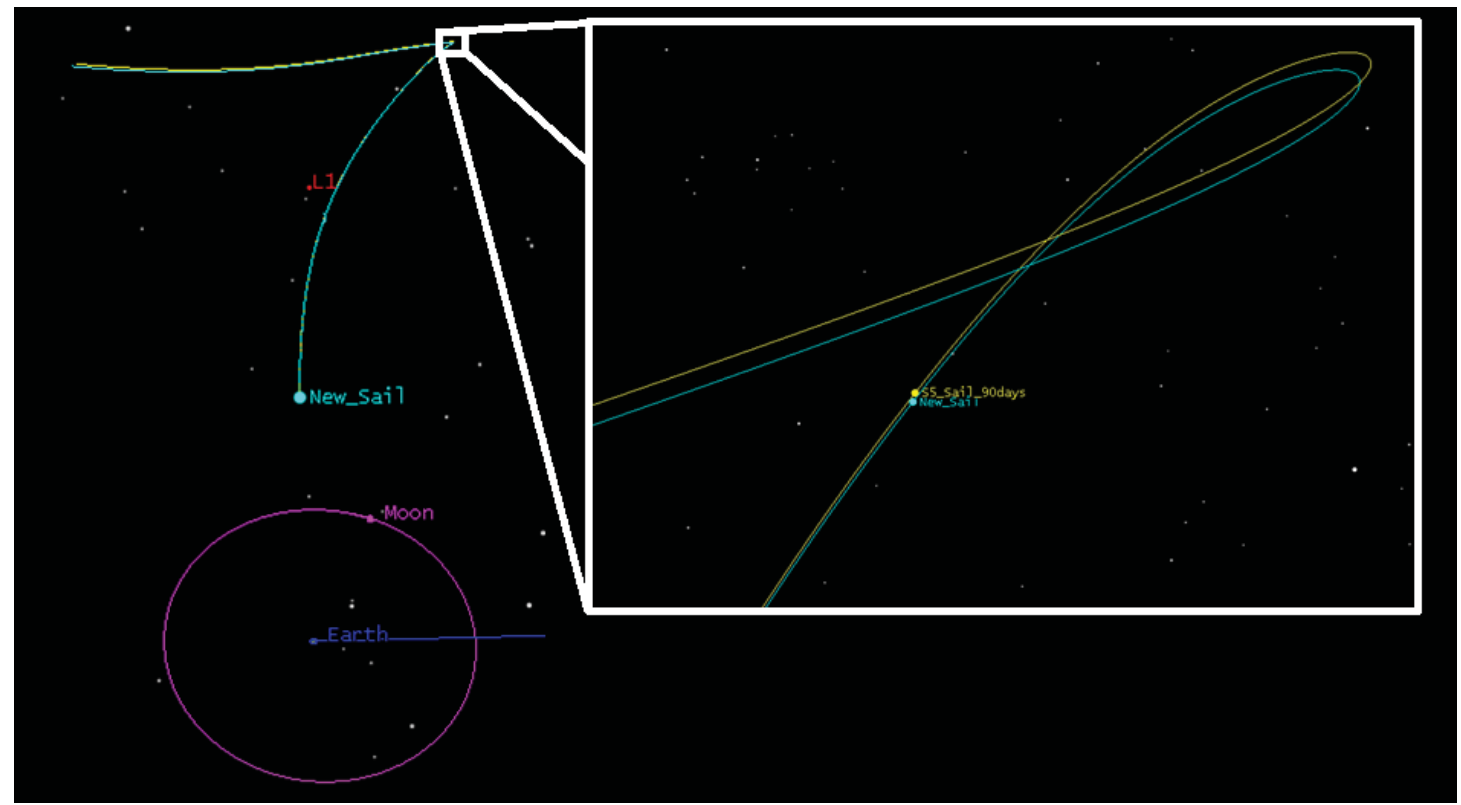




\section{Figure 3. Images from STK showing the final STK (turquoise) and S5 (yellow) trajectories being compared.}

Additionally, the STK trajectory as propagated with the solar sail was compared to the S5 simulation with no SRP (no solar sail) for the 30 day period. This gave a bound on the maximum error between the simulations, assessed the overall effect of the solar sail on the trajectory, and also allowed the effects of a sail deployment failure to be estimated in terms of the percent error values (especially to compare to the conservative $20 \%$ requirement). The results are given in Table 1 in rows 2 for both methods 1 and 2 ("Without Sail"). As seen in the table, the smallest percent error value for this case is $40 \%$ on position using the first method, significantly more than the $20 \%$ requirement.

Finally, the comparison between the two simulations with SRP being modeled in both STK and S5 is given in Table 1 in row 3 for both methods as "Sail." This comparison was done with the Derbes and Lichodziejewski SRP model $^{10}$ in S5 to match the SRP model in STK. It resulted in values of percent error on the order of $\sim 0.1 \%$, with the largest being $0.13 \%$ error on position using method 2 . Also, the trajectories from this case were plotted in STK and can be seen in Figure 3.

The results of errors on the attitude of the sail are shown in Table 2. These are the effects on the percent error values for the navigation mission requirement, and are all relative to $0 \%$ error at the trim conditions. Here, it can be seen that variance in the sun incidence angle had a much greater effect on navigation error than variation of the top spin angle. This is expected as changing the sun incidence angle affects a larger force component than changing the top spin angle because of the trim orientation of the sail.

Table 2. Results of percent error from $\pm 2^{\circ}$ variation of top spin and sun incidence angles.

\begin{tabular}{|l|l|l|}
\hline Sun Incidence Angle & Position Percent Error & Velocity Percent Error \\
\hline $33.5^{\circ}$ & $4.2162 \%$ & $4.5155 \%$ \\
\hline $37.5^{\circ}$ & $4.1894 \%$ & $4.4581 \%$ \\
\hline Top Spin Angle & Position Percent Error & Velocity Percent Error \\
\hline $8^{\circ}$ & $0.9980 \%$ & $0.8951 \%$ \\
\hline $12^{\circ}$ & $0.9980 \%$ & $0.8952 \%$ \\
\hline
\end{tabular}

\section{B. Shaped Model Results}

As stated above, the STK model tested in the validation process was a flat plate sail using the L'Garde optics model. However as can be seen in Figure 1b, the L'Garde sail has a very non-flat shape. In order to model the sail shape, L'Garde has developed force coefficients that are similar to lift coefficients for an airplane that consist of non-dimensionalzed force coefficients indexed against sun incidence angle. Previously L'Garde has delivered two sets of these coefficients to NASA. During the In Space Solar Sail Project ${ }^{14}, \mathrm{~L}^{\prime}$ Garde delivered coefficients for a 10,000 square meter sail. Another set of coefficients was delivered for the ST9 proposal of 2006, which were developed for a 950 square meter sail. L'Garde is basing the Solar Sail Demonstration project on the ST9 coefficients, since those are closer to the 1200 square meters sail size, and also because both designs include spreader bars (while the In Space sail design did) and thus the ST9 sail shape is probably also more similar to the test sail being manufactured for the Solar Sail Demonstration project.

In order to model the shape of the L'Garde sail, we use the Generalized Solar Model (GSM) developed for S5 ${ }^{15}$. The GSSM uses tensors to model non-flat sails. In the case of the L'Garde sail, since L'Garde has produced and delivered non-dimensionalzed coefficients, Rios-Reyes developed a method of fitting the L'Garde coefficients to the GSSM tensors using a least squares fit ${ }^{16}$. Rios-Reyes converted the coefficients for both the ST9 and In Space L'Garde models. We analyzed both, but here we only report results from the converted ST9 coefficients. 
Figure 4 presents the results of the least squares fit to the coefficients. The method developed by Rios-Reyes of fitting the tensors to the force coefficients does an excellent job of matching the L'Garde force coefficients across the entire range of sun incidence angles of interest. More details of how we modeled the L'Garde shape appear in the Appendix.

The results of the S5 propagated orbit using the ST9 coefficients are presented in Table 3. These results are compared to the same trajectory that appears in Table 1, which is the STK flat plate Derbes and Lichodziejewski SRP model. The larger deviation from the STK flat plate model with the L'Garde shape model is to be expected due to the large deviation from a flat plate of the L'Garde design. Based on the fidelity of the model in the L'Garde force coefficient model, this is a fairly significant result and is published here for the first time.

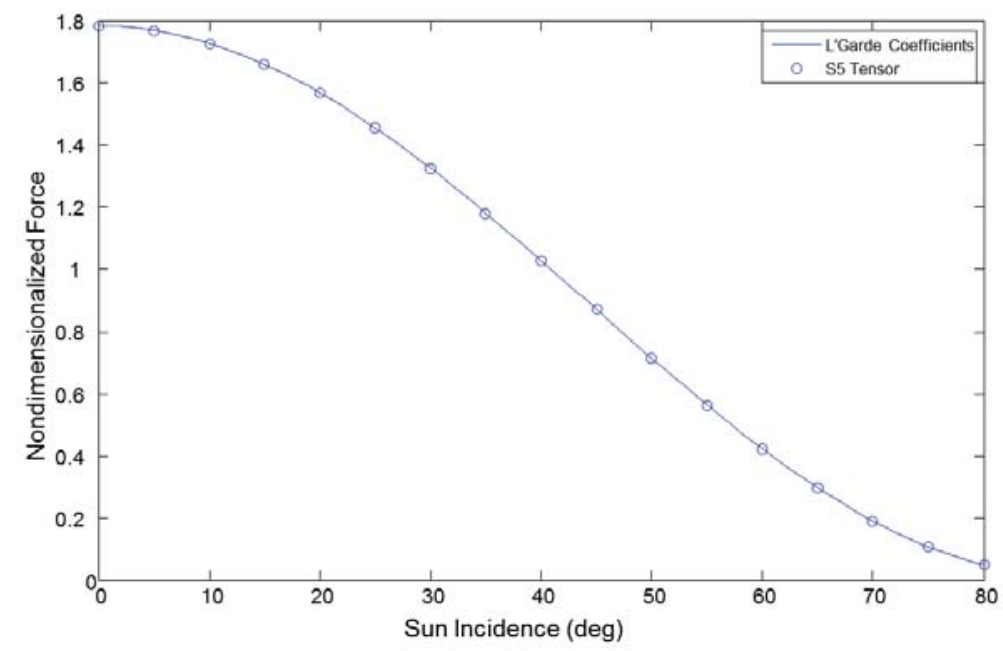

Figure 4. S5 Tensors Least Squares Fit to L'Garde Force Coefficients

Table 3. Comparison of S5 L'Garde GSM Tensor Model to L'Garde STK Flat Plate

\begin{tabular}{|l|l|l|}
\hline (Method 1) & Position Percent Error & Velocity Percent Error \\
\hline Shape to Flat Plate & $3.5327 \%$ & $16.5435 \%$ \\
\hline (Method 2 $\Delta \mathrm{r}, \Delta \mathrm{v})$ & Position Percent Error & Velocity Percent Error \\
\hline Shape to Flat Plate & $5.5657 \%$ & $5.4311 \%$ \\
\hline
\end{tabular}

Currently a shape model for STK of the L'Garde sail based on the ST9 coefficients is under development. However, those results were not available at publication time and so a comparison of the S5 shape model to the STK model remains future work.

\section{Conclusions}

The focus of this work was to validate the L'Garde STK solar sail simulation and assess the effect of the non-flat shape of the L'Garde sail on meeting mission requirements. The NASA-developed simulation S5 matched the L'Garde STK simulation of the Sunjammer 30-day maneuver sequence very closely for the flat plate model, with percent error values on the order of $\sim 0.1 \%$ on position and velocity using both methods. The degree to which the simulations match can be seen by observing that these errors are not much larger than those associated with the comparison without the sail. This shows good agreement between the sail models, but with some differences still remaining. While the difference between the two software tools could be improved to be even more accurate, these results indicate that the L'Garde simulation is accurate enough to be used for the mission requirements. Further 
refinements may be introduced at the Flight Readiness Review (FRR) phase of a future mission, and as the L'Garde simulation is updated and finalized, the existing S5 model can also be modified to continue to validate that the STK simulation is accurate. Additionally, the result of a minimum of $40 \%$ error in the comparison of the sail model in STK to the simulation with no SRP (no sail) in S5 implies that while the $20 \%$ requirement is not exceedingly demanding, it has been determined that this requirement cannot be met if the sail fails to deploy.

The attitude error study shows that the $2 \%$ requirement to point the sail will not cause the position requirement of $20 \%$ to fail even with the maximum error. The maximum deflections from trim for each angle were studied, with results indicating that errors in attitude will have a significant effect on the navigation requirement, and also that as expected, errors in the sun incidence angle have a greater effect than errors in the top spin angle.

The results of the shaped model comparison show that the shape of the L'Garde sail, as expected, has a large effect on the trajectory. This result indicates that any mission plans with a L'Garde sail, or a sail having a similar large deviation in shape from a flat plate, should incorporate a shape model for accuracy. The results also imply that calibration of a non-flat sail early in a future mission will be important, since the differences between the model and actual thrust are likely to be greater for a sail that deviates significantly from a flat plate.

The second error method is recommended for use when comparing the L'Garde simulation to the as-flown trajectory, as it resulted in values of percent error that are more consistent between velocity and position. This recommendation has been passed to the Solar Sail Demonstration Project office. A more complex analysis of the orbit propagation errors could have been performed, but the project requirement was written as a percent deviation in the final position of the sailcraft, so the analysis was performed on that basis.

Future work could extend the analysis to a comparison of the shape model being developed for STK and also other shaped models that publish results. For the attitude errors, a statistical investigation of the effects of combined error (varying both top spin and sun incidence together) on the attitude of the sail could provide valuable information for use during the comparison of the L'Garde simulation to the as-flown trajectory. A more complex analysis of the trajectory errors could also be performed, perhaps incorporating a typical covariance matrix for a notional navigation filter. A significant finding implied by the shape model results is that even flat solar sails need to consider modeling things such as boom bending or areal differences between sail quadrants as a sanity check on the flat plate model. This work will help inform future missions for the L'Garde sail as well as other current sail projects such as the NASA NEA Scout and Lunar Flashlight missions, the Surrey Cubesail, or the Planetary Society Lightsail. 


\section{Appendix}

The constants from the SRP force model developed by Derbes and Lichodziejewski. ${ }^{10}$ This is the model used by STK for the flat plate comparison.

$R_{p}=0.85268583078932$

$\theta_{p}=25.9315208633397$

$\rho_{d}=0.894100805958922$

$\varepsilon_{f}=0.05$

$\varepsilon_{b}=0.62$

$B_{f}=2 / 3$

$B_{b}=2 / 3$

For the modification to the sine term in Equation (3) we used:

$\mathrm{k}=1.037260834533588$ (i.e., $\theta_{p}=k \theta_{i}$ for the sine term)

For the shape model in section IV, we used the following equation which is Rios-Reyes ${ }^{16}$ Equation (15):

$$
\mathbf{F}_{c}=\left[\begin{array}{ccccc}
a_{2} \hat{r}_{1} & 0 & -4 \rho s \hat{r}_{1} \hat{r}_{3} & 0 & -a_{3} \hat{r}_{1} \hat{r}_{3} \\
0 & a_{2} \hat{r}_{3} & -2 \rho s \hat{r}_{1}^{2} & -2 \rho s \hat{r}_{3}^{2} & -a_{3} \hat{r}_{3}^{2}
\end{array}\right]\left[\begin{array}{c}
J_{11}^{2} \\
J_{33}^{2} \\
J_{113}^{3} \\
J_{333}^{3} \\
J_{3}^{1}
\end{array}\right]
$$

For which the tensors are:

$J_{11}^{2}=0.1172$

$J_{33}^{2}=2.9733$

$J_{113}^{3}=0.0042$

$J_{333}^{3}=1.1347$

$J_{3}^{1}=-1.1079$

The above tensors were matched to the L'Garde force coefficients using a Least Squares Fit by Rios-Reyes ${ }^{16}$.

And the optical coefficients are (McInnes model):

$s=1.0000$

$\rho=0.8941008$

$\varepsilon_{f}=0.05$

$\varepsilon_{b}=0.40$

$B_{f}=0.79$

$B_{b}=0.55$

The above coefficients are what Rios-Reyes fit the tensors to, and are his approximation of the Derbes model. These coefficients are also reduced by Rios-Reyes to:

$\alpha_{2}=B_{f}(1-s) \rho+(1-\rho)\left[\frac{\epsilon_{f} B_{f}-\epsilon_{b} B_{b}}{\epsilon_{f}-\epsilon_{b}}\right]=-0.04248$

$\alpha_{3}=1-\rho s=0.10590$

$\hat{r}_{1}, \hat{r}_{2}, \hat{r}_{3}$ represent the sail to sun vector in the body frame of the sail where the 3 - axis is perpendicular to the normal of the sail surface (see Figure 2). 


\section{Acknowledgments}

Alex Braafladt was supported by the Minnesota Space Grant Consortium, a part of the National Space Grant College and Fellowship Program. Alexandra Artusio-Glimpse was supported by the National Science Foundation. Andrew Heaton was supported in part by the Solar Sail Demonstration Project and in part by the NASA Office of Chief Technologist.

\section{References}

${ }^{1}$ McInnes, C. R., Solar Sailing: Technology, Dynamics, and Mission Applications, Springer-Praxis Series in Space Science and Technology, Springer-Praxis, Chichester, UK, 1999.

${ }^{2}$ Wright, J. L., Space Sailing, Gordon and Breach Science Publishers, 1992.

${ }^{3}$ Murray, B., Flight to Mercury, Columbia University Press, 1977.

${ }^{4}$ Murphy, D., "Validation of A Scalable Solar Sailcraft," 53 ${ }^{\text {rd }}$ JANNAF Propulsion Meeting, December 2005.

${ }^{5}$ Lichodziejewski, D., et al., "Vacuum Deployment and Testing of a 20-m Solar Sail System," $47^{\text {th }}$

AIAA/ASME/ASCE/AHS/ASC Structures, Structural Dynamics, and Materials Conference, May 2006, AIAA 20061705.

${ }^{6}$ Alhorn, D., et al., "NanoSail-D: The Small Satellite That Could!" AIAA/USC Conference on Small Satellites, August 2011.

${ }^{7}$ O’Shaughnessy, D. J., McAdams, J. V., Williams, K. E., and Page, B. R., "Fire Sail: MESSENGER's Use of Solar Radiation Pressure for Accurate Mercury Flybys," $32^{\text {nd }}$ Guidance and Control Conference, American Astronautical Society, February 2009, AAS 09-014.

${ }^{8}$ Sawada, H., et al., "Mission Report on The Solar Power Sail Deployment Demonstration of IKAROS," $52^{\text {nd }}$ AIAA/ASME/ASCE/AHS/ASC Structures, Structural Dynamics, and Materials Conference, 2011, AIAA 2011-1887.

${ }^{9}$ Ellis, J., Lisano, M., Wolff, P., Evans, J., Bladt, J., Scheeres, D., Rios-Reyes, L., and Lawrence, D., “A Solar Sail Integrated Simulation Toolkit," $14^{\text {th }}$ AAS/AIAA Space Flight Mechanics Conference, 2004, AAS 04-283.

${ }^{10}$ Derbes, B., and Lichodziejewski, D., "Propulsive Reflectivity and Photoflexibility: Effects on Solar Sail Performance and Control," 42 ${ }^{\text {nd }}$ AIAA/ASME/SAE/ASEE Joint Propulsion Conference \& Exhibit, July 9, AIAA 2006-4520.

${ }^{11}$ Derbes, B., "Format and Math Specifications for Reporting Propulsive Performance of Square Solar Sails," L'Garde Technical Report LTR-04-BD-003, Tustin, CA, 2005.

${ }^{12} \mathrm{Ma}, \mathrm{C}$., and Feissel, M., "Definition and Realization of the International Celestial Reference System by VLBI Astrometry of Extragalactic Objects," Central Bureau of IERS, Paris, France, June 1997, IERS Technical Note 23.

${ }^{13}$ Curtis, H. D., Orbital Mechanics for Engineering Students, $2^{\text {nd }}$ ed., Elsevier Aerospace Engineering Series, Butterworth-Heinemann, Burlington, MA, 2010.

${ }^{14}$ Garbe, G., and Montgomery, E., “An Overview of NASA’s Solar Sail Propulsion Project,” AIAA Paper 20034662, July 2003.

${ }^{15}$ Rios-Reyes, L., Scheeres, D., "Generalized Model for Solar Sails”, Journal of Spacecraft and Rockets, Vol. 42 , No. 1, 2005.

${ }^{16}$ Rios-Reyes, L., Scheeres, D., Comparison Between L'Garde's Force Coefficients Model and the Generalized Sail Model", Internal NASA Memo, January 2005. 